ANNALES

POLONICI MATHEMATICI

$86.3(2005)$

\title{
Courants algébriques et courants de Liouville
}

\author{
par M. Blel (Monastir), S. K. Mimouni (Monastir) \\ et G. RABY (Poitiers)
}

\begin{abstract}
We define in $\mathbb{C}^{n}$ the concepts of algebraic currents and Liouville currents, thus extending the concepts of algebraic complex subsets and Liouville subsets. After having shown that every algebraic current is Liouville, we characterize those positive closed currents on $\mathbb{C}^{n}$ which are algebraic. Let $T$ be a closed positive current on $\mathbb{C}^{n}$. We give sufficient conditions, relating to the growth of the projective mass of $T$, so that $T$ is Liouville. These results generalize those previously obtained by N. Sibony and P. M. Wong, and $\mathrm{K}$. Takegoshi in the geometrical case, i.e. when $T=[X]$ is the current of integration on an analytical complex subset of $\mathbb{C}^{n}$.
\end{abstract}

Introduction. Un sous-ensemble analytique complexe de $\mathbb{C}^{n}$ est un espace de Liouville si ses seules fonctions holomorphes bornées sont les constantes. $\mathbb{C}^{n}$ est donc un espace de Liouville ainsi que, plus généralement, un sous-ensemble algébrique complexe de $\mathbb{C}^{n}$ et le graphe $\{(z, w) ; w=f(z)$, $z \in X\}$ défini par une fonction holomorphe sur une sous-variété algébrique complexe $X$ de $\mathbb{C}^{n}$. De nombreux auteurs ont donné des conditions suffisantes pour qu'un sous-ensemble analytique complexe de $\mathbb{C}^{n}$ soit de Liouville, par exemple [KA96], [RO80],[SI-WO81], [TA93], [TO96]. Le problème de la caractérisation des espaces de Liouville reste ouvert, alors qu'un sousensemble algébrique de $\mathbb{C}^{n}$ se caractérise par une propriété de croissance du volume de sa trace sur la boule $B(r)$ centrée en 0 et de rayon $r$ (cf. [ST64]). Plus précisément, un sous-ensemble analytique complexe $X$ de $\mathbb{C}^{n}$, de dimension $p$, est algébrique si et seulement si il existe une constante $C>0$ telle que

$$
\operatorname{Vol}(X \cap B(r)) \leq C r^{2 p} \quad \text { pour tout } r>0 .
$$

2000 Mathematics Subject Classification: 32C25, 32C30, 12D10, 14A20.

Key words and phrases: plurisubharmonic function, positive current, complex analytic set, algebraic set, Liouville property.

Ce travail a bénéficié de l'aide du CMCU dans le cadre des actions intégrées (France Tunisie).

Supported by College of Science-Research Center (KSU) project N (Math/2005/11). 
Dans cet article nous étendons ces notions aux courants positifs fermés en définissant les courants algébriques et les courants de Liouville. Nous généralisons à ces types de courants les résultats connus dans le cas géométrique, c'est-à-dire lorsque $T=[X]$ est le courant d'intégration sur un sousensemble analytique complexe $X$ de $\mathbb{C}^{n}$.

Ainsi, dans la section 2 , si $T$ est un courant positif fermé sur $\mathbb{C}^{n}$, de dimension $p$, on dit que $T$ est algébrique s'il existe une constante $C>0$ telle que

$$
\sigma_{T}(B(r)) \leq C r^{2 p} \quad \text { pour tout } r>0,
$$

où $\sigma_{T}=T \wedge \frac{1}{p !}\left(i \partial \bar{\partial}|z|^{2}\right)^{p}$ est la mesure trace du courant $T$. Nous caractérisons ces courants algébriques par l'algébricité de leurs tranches sur les hyperplans, d'une part par tranches concourantes et d'autres part par tranches parallèles aux hyperplans de coordonnées.

La notion de courant de Liouville est introduite dans la section 3. Si $T$ est un courant positif fermé sur $\mathbb{C}^{n}$, on dit que $T$ est de Liouville si pour toute fonction $f$ holomorphe sur $\mathbb{C}^{n}$ et bornée sur le support de $T$, on a $\partial \bar{\partial}\left(|f|^{2} T\right)=0$. On montre alors que tout courant algébrique est de Liouville. Plus précisément, on démontre que si $T$ est un courant positif fermé sur $\mathbb{C}^{n}$ vérifiant

$$
\varliminf_{R \rightarrow+\infty} \frac{1}{(\log R)^{2}} \int_{1}^{R} \frac{\sigma_{T}(B(r))}{r^{2 p+1}} d r=0,
$$

alors toute fonction $u$ plurisousharmonique et bornée sur le support de $T$ vérifie $\partial \bar{\partial}(u T)=0$.

Pour l'essentiel, la section 4 est consacrée à la généralisation d'un théorème de K. Takegoshi ([TA93]). En particulier on montre que si $T$ est un courant positif fermé de dimension $p$ sur $\mathbb{C}^{n}$, vérifiant

$$
\int_{1}^{+\infty} \frac{d r}{r \nu_{T}(r)}=+\infty \quad \text { où } \quad \nu_{T}(r)=\frac{\sigma_{T}(B(r))}{\tau_{p} r^{2 p}}
$$

$\left(\tau_{p}\right.$ désignant le volume de la boule unité de $\left.\mathbb{C}^{p}\right)$, alors $T$ est de Liouville. La condition suffisante obtenue n'est pas nécessaire comme l'illustre l'exemple où $T$ est le courant d'intégration sur l'ensemble de Liouville défini par $\left\{(z, w) \in \mathbb{C}^{2} ; w=e^{-i z^{2}}\right\}$.

La fin de cet article est dédiée à la caractérisation des courants positifs fermés dans $\mathbb{C}^{n}$, de dimension $n-1$, à support contenu dans $\{|P|<C\}$, où $P$ est un polynôme non constant sur $\mathbb{C}^{n}$. Nous montrons que ces courants sont algébriques et obtenus comme moyenne de courants d'intégrations sur des sous-ensembles algébriques.

Les résultats des sections 4 et 5 ont été annoncés dans [MI00]. Les auteurs remercient J.-P. Demailly pour l'aide et les conseils qu'il a apportés au cours de l'élaboration de ce travail. 
1. Notations. Soit $\Omega$ un ouvert de $\mathbb{C}^{n}$. On considère les opérateurs $d=\partial+\bar{\partial}$ et $d^{c}=(i / 2)(\bar{\partial}+\partial)$ de sorte que $d d^{c}=i \partial \bar{\partial}$. Soit $\beta=(i / 2) \partial \bar{\partial}|z|^{2}$ la forme de Kähler sur $\mathbb{C}^{n}$ et $\alpha=i \partial \bar{\partial} \log |z|$. On note $\mathcal{D}_{(p, q)}(\Omega)$ l'espace des formes différentielles de bidegré $(p, q)$ de classe $\mathcal{C}^{\infty}$ à support compact dans $\Omega$. On munit cet espace de la topologie usuelle de la convergence uniforme sur tout compact de toutes les dérivées des coefficients. L'espace des formes linéaires continues sur $\mathcal{D}_{(p, q)}(\Omega)$, noté $\mathcal{D}_{(p, q)}^{\prime}(\Omega)$, est appelé l'espace des courants de bidimension $(p, q)$ sur $\Omega$. Soit $T \in \mathcal{D}_{(p, q)}^{\prime}(\Omega)$; alors $T$ s'écrit canoniquement sous la forme

$$
T=\sum_{|I|=n-p,|J|=n-q} T_{I, J} \frac{i^{(n-p)(n-q)}}{2^{(p+q) / 2}} d z_{I} \wedge d \bar{z}_{J},
$$

où $T_{I, J}$ sont des distributions et la somme est étendue à tous les multi-indices $I$ et $J$ strictement croissants. Si $p=q$ on pose $\sigma_{p}=i^{p^{2}} / 2^{p}$.

Un courant $T \in \mathcal{D}_{(p, p)}^{\prime}$ est dit positif si pour toutes formes $\alpha_{1}, \ldots, \alpha_{p}$ de classe $\mathcal{C}^{\infty}$ et de bidegré $(1,0)$,

$$
T \wedge i \alpha_{1} \wedge \bar{\alpha}_{1} \wedge \cdots \wedge i \alpha_{p} \wedge \bar{\alpha}_{p}
$$

est une mesure positive.

On appelle mesure trace d'un courant positif fermé $T$ de bidimension $(p, p)$ la mesure de Radon positive

$$
\sigma_{T}=T \wedge \frac{\beta^{p}}{p !}
$$

Si $T=\sum_{|I|=|J|=n-p} T_{I, J} \sigma_{p} d z_{I} \wedge d \bar{z}_{J}$, alors $\sigma_{T}=\left(\sum_{|I|=n-p} T_{I, I}\right) \beta^{n} / n$ !. On note $\sigma_{T}(r)=\sigma_{T}(B(0, r))$ et $\nu_{T}(r)=\sigma_{T}(r) / \tau_{p} r^{2 p}$, avec $\tau_{p}=\pi^{p} / p$ !, le volume de la boule unité de $\mathbb{C}^{p}$. On rappelle la formule de Lelong-Jensen pour un courant positif : si $R>r$ on a

$$
\nu_{T}(R)-\nu_{T}(r)=\int_{B(0, R) \backslash B(0, r)} T \wedge \alpha^{p} .
$$

Il en résulte que la fonction $\nu_{T}(r)$ est croissante en $r$. La limite $\lim _{r \rightarrow 0} \nu_{T}(r)$ est appelée le nombre de Lelong du courant $T$ au point 0 (cf. [LE-GR86]).

\section{Courants algébriques}

DÉfinition 2.1. Un courant positif fermé $T$ sur $\mathbb{C}^{n}$ de bidimension $(p, p)$ est dit algébrique, s'il existe une constante $C>0$ telle que

$$
\nu_{T}(r)=\frac{\sigma_{T}(r)}{\tau_{p} r^{2 p}}=\frac{1}{\pi^{p} r^{2 p}} \int_{B(0, r)} T \wedge \beta^{p} \leq C \quad \forall r>0 .
$$

Exemple. Si $P$ est un polynôme non nul sur $\mathbb{C}^{n}$, alors le courant $(i / \pi) \partial \bar{\partial} \log |P|$ est algébrique. 


\section{REMARques.}

1. Dans le cas où $T=[X]$ est le courant d'intégration sur un ensemble analytique $X$ de $\mathbb{C}^{n}$, W. Stoll [ST64] a démontré que l'ensemble analytique $X$ est algébrique ssi le courant $T$ est algébrique.

2. Comme l'application $R \mapsto \nu_{T}(R)=\sigma_{T}(B(0, R)) / \tau_{p} R^{2 p}$ est croissante, elle admet une limite quand $R$ tend vers $+\infty$. Cette limite est appelée le degré d'algébricité du courant $T$ et sera notée $\delta(T)$.

3. Il résulte de la formule de Lelong-Jensen que si $T$ est un courant positif fermé de bidimension $(p, p)$ algébrique, alors $\int_{\mathbb{C}^{n}} T \wedge \alpha^{p}<$ $+\infty$. Alors, d'après le théorème de Skoda-El Mir [SK82], [EL84], le courant se prolonge en un courant positif fermé sur $\mathbb{P}^{n}$. On note $\widetilde{T}$ ce prolongement. Si $\pi: \mathbb{C}^{n+1} \backslash\{0\} \rightarrow \mathbb{P}^{n}$ est la projection canonique, alors le courant $\pi^{*}(\widetilde{T})$ est un courant positif fermé de bidimension $(p+1, p+1)$ sur $\mathbb{C}^{n+1} \backslash\{0\}$ et se prolonge en un courant positif fermé $\Theta$ sur $\mathbb{C}^{n+1}$. Le courant $\Theta$ est un courant conique, i.e. $h_{R}^{*} \Theta=\Theta$ pour tout $R \in \mathbb{C}^{*}$, avec $h_{R}$ l'homothétie de centre l'origine et de rapport $R$ $\left(h_{R}(z)=R z\right)$.

4. Dans le cas $p=n-1$ (cf. [LE-GR86], [BL89], [BL92], [FO-SI95]) on a les équivalences suivantes:

(i) $\theta$ est un courant conique de bidegré $(1,1)$;

(ii) il existe une fonction $v \in \operatorname{Psh}\left(\mathbb{C}^{n}\right)$ telle que $\theta=d d^{c} v$ et $v(\lambda z)=$ $v(z)+c \log |\lambda|$ pour tous $\lambda \in \mathbb{C}^{*}$ et $z \in \mathbb{C}^{n}\left(c=\nu_{\theta}(0)\right)$.

On rappelle le lemme suivant (cf. [BL-DE-MO90]) qui caractérise les courants coniques.

Lemme 2.2. Soit $\Theta$ un courant positif fermé de bidimension $(p, p)$ sur $\mathbb{C}^{n}$ où $1 \leq p \leq n-1$. Alors il y a équivalence entre les quatre propriétés suivantes:

(a) $\Theta$ est invariant par les homothéties $h_{a}$ de rapport $a \in \mathbb{C}^{\star}$.

(b) $\Theta$ est invariant par les homothéties $h_{r}$ de rapport $r>0$.

(c) $\Theta \wedge \alpha^{p}=0$ sur $\mathbb{C}^{n} \backslash\{0\}$.

(d) $\Theta$ est l'extension à $\mathbb{C}^{n}$ de l'image réciproque d'un courant positif fermé $\theta$ sur $\mathbb{P}^{n-1}$ par la projection $\pi: \mathbb{C}^{n} \backslash\{0\} \rightarrow \mathbb{P}^{n-1}$.

Lemme 2.3. Si $T$ est un courant positif fermé de bidimension $(p, p)$ sur $\mathbb{C}^{n}$, on définit la fonction de comptage $N_{T}$ par

$$
N_{T}(r)=\int_{1}^{r} \frac{\nu_{T}(t)}{t} d t .
$$

Alors on a les équivalences suivantes: 
(i) T est algébrique.

(ii) $\nu_{T}(r)=O(1)$.

(iii) $N_{T}(r)=O(\log (r))$.

La démonstration est élémentaire.

Algébricité et tranchage (slicing). On rappelle d'abord la définition de la tranche d'un courant positif fermé sur une hypersurface (cf. [RA96]). Pour le tranchage dans un cadre plus général, on renvoi le lecteur à [BM-EM97]. Si $T$ est un courant positif fermé sur $\mathbb{C}^{n}$ et $L$ l'hypersurface définie par $L=\left\{z_{1}=0\right\}$, la tranche (ou le slice) de $T$ sur $L$, notée $T \uparrow_{L}$, est définie si la limite existe par

$$
\left\langle T \uparrow_{L}, \varphi\right\rangle=\lim _{\varepsilon \rightarrow 0} \frac{1}{\pi \varepsilon^{2}}\left\langle T, \varphi \wedge \frac{i}{2} d z_{1} \wedge d \bar{z}_{1} \chi_{\left\{\left|z_{1}\right|<\varepsilon\right\}}\right\rangle,
$$

où $\chi_{\left\{\left|z_{1}\right|<\varepsilon\right\}}$ désigne la fonction caractéristique du cylindre $\left\{\left|z_{1}\right|<\varepsilon\right\}$ et $\varphi \in$ $\mathcal{D}_{p-1, p-1}\left(\mathbb{C}^{n}\right)$. De plus, à $\omega \in \mathbb{P}^{n-1}$, on associe l'hyperplan $L^{\omega}=\left\{f_{\omega}=0\right\}$ avec $f_{\omega}(z)=\omega \cdot z /\|\omega\|$.

La tranche $T \uparrow_{L^{\omega}}$ existe sauf sur un ensemble $A \subset \mathbb{P}^{n-1}$ pluripolaire [RA96]; elle est définie par

$$
T \uparrow_{L^{\omega}}=d d^{c}\left(\log \left|f_{\omega}\right| T\right) .
$$

On se propose de mettre en évidence le lien entre l'algébricité d'un courant positif fermé et l'algébricité de ses tranches sur les hypersurfaces.

THÉORÈmE 2.4. Soit T un courant positif fermé sur $\mathbb{C}^{n}$ de bidimension $(p, p)$ avec $1 \leq p \leq n-1$. S'il existe un sous-ensemble A non pluripolaire de la grassmannienne $G_{n-1, n}$ tel que la tranche de $T$ sur chaque hyperplan $L$ de $A$ est algébrique, alors le courant $T$ est algébrique.

Corollaire 2.5. Soit $T \in \mathcal{D}_{n-1, n-1}^{\prime}\left(\mathbb{C}^{n}\right)$ un courant positif fermé. On suppose qu'il existe un polynôme $P$ non constant et une constante $C>0$ tels que $\operatorname{Supp} T \subset\{z ;|P(z)|<C\}$. Alors $T$ est algébrique.

Dans le cas où $T$ est le courant d'intégration sur un ensemble analytique $X$, on retrouve le résultat de Togni [TO96]. On donnera dans la section 5 une caractérisation de ces courants qu'on appellera courants à support tubulaire.

Démonstration du corollaire 2.5. Il existe un sous-ensemble $A$ pluripolaire de $\mathbb{P}^{n-1}$ tel que la tranche $T \uparrow_{L^{\omega}}$ de $T$ sur $L^{\omega}$ existe pour $\omega \notin A$ (cf. [RA96], [BM-EM97]). On pose $A^{\prime}=A \cup\left\{\omega ; P \uparrow_{L^{\omega}}=\right.$ const $\}$. Alors $A^{\prime}$ est encore pluripolaire et pour $\omega \notin A^{\prime}, T \uparrow_{L^{\omega}}$ est un courant positif fermé et $\operatorname{Supp}\left(T \uparrow_{L^{\omega}}\right) \subset\left\{z \in L^{\omega} ;|P(z)|<C\right\}$. Donc par récurrence on se ramène au cas $n=1$ qui est alors évident puisque dans ce cas $\mu=T$ est une mesure positive à support compact. 
On rappelle la formule de Croffton (cf. [SI74]) : si $S$ est un courant positif fermé de bidimension $(1,1)$ sur $\mathbb{C}^{n}$, alors

$$
\nu_{S}(r)=\frac{\sigma_{S}(r)}{\pi r^{2}}=\int_{\mathbb{P}^{n-1}}\left(\int_{L^{\omega} \cap B(0, r)} S \uparrow_{L^{\omega}}\right) d \omega .
$$

En appliquant cette formule au courant $S=T \wedge \alpha^{p-1}$ on aura

$$
N_{T}(r)=\int_{\mathbb{P}^{n-1}} N_{\left.T\right|_{L^{\omega}}}(r) d \omega .
$$

Pour la suite de la démonstration du théorème 2.4 on généralise le résultat de Molzon-Shifman-Sibony [MO-SH-SI81] aux courants positifs fermés de $\mathbb{C}^{n}$.

LeMme 2.6. Soit $T$ un courant positif fermé de bidimension $(p, p)$ sur $\mathbb{C}^{n}, p \geq 1$. Si $\mu$ est une mesure de probabilité sur $\mathbb{P}^{n-1}=G_{(n-1, n)}$, on notera $U$ le potentiel logarithmique de $\mu$ défini par

$$
U(z)=\int_{\mathbb{P}^{n-1}} K(z, \omega) d \mu(w) \text { avec } K(z, \omega)=\log \frac{|z||\omega|}{|z \cdot \omega|} .
$$

On suppose que $U \leq C<+\infty$. Alors, pour tout entier $k \in \mathbb{N}^{*}$ il existe deux constantes $C_{1}>0, a_{k}>0$ telles que pour tout $r>0$,

$$
C_{1} N_{T}\left(\frac{e^{-2 C} r}{2}\right) \leq \int_{\mathbb{P}^{n-1}} N_{T \uparrow_{L} \omega}(r) d \mu(\omega) \leq a_{k} N_{T}(k r), \quad a_{k}=O\left(\log \left(\frac{1}{k}\right)\right) .
$$

Démonstration. Rappelons que $L^{\omega}$ est l'hyperplan de $\mathbb{C}^{n}$ passant par 0 et défini par $f_{\omega}=0$ avec $f_{\omega}(z)=\omega . z /\|\omega\|, \omega \in \mathbb{P}^{n-1}$. Il résulte des travaux de G. Raby que $\left.T\right|_{L^{\omega}}=d d^{c}\left(\log \left|f_{\omega}\right| T\right)$ sauf pour $\omega$ dans un sous-ensemble pluripolaire de $\mathbb{P}^{n-1}$.

Soit $k>1$. On note

$$
\mu_{k}=\int_{U(n)} \psi_{1 / k}(\mathfrak{g})\left(\mathfrak{g}_{*} \mu\right) d \mathfrak{g}
$$

une régularisation de $\mu$, avec $U(n)$ le groupe unitaire dans $\mathbb{C}^{n}$. On note $U_{k}$ le potentiel de $\mu_{k}$. Il résulte des hypothèses faites sur la mesure $\mu$ que $0 \leq U \leq C$ et $0 \leq U_{k} \leq C$ pour tout $k \in \mathbb{N}^{*}$. On pose $V_{k}=\log |z|-U_{k}$. On a

$$
V_{k}(z)=\int_{\mathbb{P}^{n-1}} \log \left|f_{\omega}\right| d \mu_{k}(\omega)
$$

Alors

$$
\begin{aligned}
\pi^{p-1} \int_{\mathbb{P}^{n-1}} \nu_{T \Gamma_{L} \omega}(r) & d \mu_{k}(\omega) \\
& =\int_{\mathbb{P}^{n-1}} \frac{1}{r^{2 p-2}}\left(\int_{B(0, r)} d d^{c}\left(\log \left|f_{\omega}\right| T\right) \wedge \beta^{p-1}\right) d \mu_{k}(\omega)
\end{aligned}
$$




$$
\begin{aligned}
& =\frac{1}{r^{2 p-2}} \int_{B(0, r)} d d^{c}\left(\int_{\mathbb{P}^{n-1}} \log \left|f_{\omega}\right| d \mu_{k}(\omega)\right) T \wedge \beta^{p-1} \\
& =\frac{1}{r^{2 p-2}} \int_{B(0, r)} d d^{c} V_{k} \wedge T \wedge \beta^{p-1} .
\end{aligned}
$$

On a $\log |z|-C \leq V_{k}(z) \leq \log |z|$. On pose $A(r)=\left\{z ; V_{k}(z) \leq \log r\right\}$. Il en résulte que $B(0, r) \subset A(r) \subset B\left(0, e^{C} r\right)$.

Si on pose

$$
t(r)=\sup _{z \in \operatorname{Supp} T \cap A(r)}|z|^{2}, \quad I(r)=\frac{1}{r^{2 p-2}} \int_{B(0, r)} d d^{c} V_{k} \wedge T \wedge \beta^{p-1},
$$

on a $t(r) \leq e^{2 C} r^{2}$ et

$$
\begin{aligned}
I(r) & \geq \frac{e^{-2 C} t(r)}{r^{2 p}} \int_{A\left(r / e^{C}\right)} d d^{c} V_{k} \wedge T \wedge \beta^{p-1} \\
& =\frac{e^{-2 C} t(r)}{r^{2 p}} \int_{\partial A\left(r / e^{C}\right)} d^{c} V_{k} \wedge T \wedge \beta^{p-1} \\
& \geq \frac{e^{-2 C}}{r^{2 p}} \int_{\partial A\left(r e^{-C}\right)}|z|^{2} d^{c} V_{k} \wedge T \wedge \beta^{p-1} \\
& =\frac{e^{-2 C}}{r^{2 p}} \int_{A\left(r / e^{C}\right)} d V_{k} \wedge d^{c}|z|^{2} \wedge T \wedge \beta^{p-1}+\frac{e^{-2 C}}{r^{2 p}} \int_{A\left(r / e^{C}\right)}|z|^{2} d d^{c} V_{k} \wedge T \wedge \beta^{p-1} \\
& \geq \frac{e^{-2 C}}{r^{2 p}} \int_{\partial A\left(r / e^{C}\right)} V_{k} d^{c}|z|^{2} \wedge T \wedge \beta^{p-1}-\frac{e^{-2 C}}{r^{2 p}} \int_{A\left(r / e^{C}\right)} V_{k} T \wedge \beta^{p} \\
& \geq \frac{e^{-2 C}}{r^{2 p}} \int_{A\left(r e^{-C} / 2\right)}\left(\log r e^{-C}-V_{k}\right) T \wedge \beta^{p} \geq \frac{e^{-2 C} \log 2}{r^{2 p}} \quad \int \beta_{A\left(r / 2 e^{C}\right)} T \wedge \beta^{p} \\
& \geq \frac{e^{-2 C} \log 2}{r^{2 p}} \int_{B\left(0, r / 2 e^{C}\right)} T \wedge \beta^{p} .
\end{aligned}
$$

Il en résulte que

$$
\nu_{T}\left(\frac{r e^{-C}}{2}\right) \leq \int_{\mathbb{P}^{n-1}} \nu_{T \uparrow_{L} \omega}(r) d \mu_{k}(\omega), \quad N_{T}\left(\frac{r e^{-C}}{2}\right) \leq \int_{\mathbb{P}^{n-1}} N_{T \uparrow_{L} \omega}(r) d \mu_{k}(\omega) .
$$

La même inégalité reste valable pour la mesure $\mu$ en faisant tendre $k$ vers $+\infty$.

On démontre l'autre inégalité de la même manière. D'où l'algébricité de $T$. 
Remarque. Si $X$ est un ensemble algébrique de $\mathbb{C}^{2}$, alors les tranches de $X$ sur les droites complexes parallèles aux axes sont algébriques. On note $\left\langle X, \omega_{1}\right\rangle$ respectivement $\left\langle X, \omega_{2}\right\rangle$ la tranche de $X$ sur l'axe $\left\{z_{1}=\omega_{1}\right\}$ respectivement $\left\{z_{2}=\omega_{2}\right\}$. Dans [AU95], Aupetit étudie le problème inverse. Remarquons que si un sous-ensemble $X$ de $\mathbb{C}^{2}$ est fermé et tel que ces tranches $\left\langle X, \omega_{1}\right\rangle$ et $\left\langle X, \omega_{2}\right\rangle$ sont algébriques, alors $X$ n'est pas forcément un sousensemble analytique : il suffit par exemple de prendre $X=\{(z, \bar{z}) ; z \in \mathbb{C}\}$. Si on ajoute l'hypothèse que $\mathbb{C}^{2} \backslash X$ est un ouvert d'holomorphie, alors Aupetit démontre que $X$ est un sous-ensemble algébrique. La démonstration peut être simplifiée de la manière suivante. Dans [AU95], le premier lemme montre dans ce cas que $X$ est un sous-ensemble analytique dont la masse totale des tranches $\left\langle X, z_{1}\right\rangle$ et $\left\langle X, z_{2}\right\rangle$ est bornée indépendamment de $z_{1}$ et $z_{2}$ par une constante $M$, en dehors d'un ensemble fini. Alors si on utilise la formule de tranchage [BM-EM97], on aura

$$
\begin{aligned}
\frac{1}{R^{2}} \int_{B(0, R)}[X] \wedge \beta \leq & \frac{1}{R^{2}} \int_{D(0, R)}\left\|\left\langle X, z_{1}\right\rangle\right\| i d z_{1} \wedge d \bar{z}_{1} \\
& +\frac{1}{R^{2}} \int_{D(0, R)}\left\|\left\langle X, z_{2}\right\rangle\right\| i d z_{2} \wedge d \bar{z}_{2} \leq 2 M \pi .
\end{aligned}
$$

Donc $X$ est algébrique. On se propose de généraliser ce résultat aux courants positifs fermés dans $\mathbb{C}^{n}$. On énonce :

THÉORÈme 2.7. Soit $T$ un courant positif fermé de bidimension $(1,1)$ sur $\mathbb{C}^{n}$. Soit $\pi_{j}: \mathbb{C}^{n} \rightarrow \mathbb{C}$ la projection sur la $j^{\text {ème }}$ coordonnée de $\mathbb{C}^{n}$ définie par $\pi_{j}\left(z_{1}, \ldots, z_{n}\right)=z_{j}$. On note $\left\langle T, \pi_{j}, z_{j}\right\rangle$ la tranche $d u$ courant $T$ sur $\pi_{j}^{-1}\left(z_{j}\right)$. On suppose que pour tout $1 \leq j \leq n$, il existe un compact $K_{j}$ de $\mathbb{C}$ tel que $\left\langle T, \pi_{j}, z_{j}\right\rangle$ est algébrique pour $z_{j} \notin K_{j}$. Alors le courant $T$ est algébrique.

Pour la commodité d'écriture, on fera la démonstration du théorème dans le cas $n=1$. On rappelle que la mesure $\left\langle T, \pi_{j}, z_{j}\right\rangle$ est algébrique si elle est bornée.

Pour la démonstration on rappelle le lemme suivant (cf. [BM-EM97]).

Lemme 2.8. Soit $T$ un courant positif fermé de bidimension $(p, p)$ sur un ouvert $\Omega$ de $\mathbb{C}^{n}$, avec $1 \leq p \leq n-1$. Soit $v$ une fonction plurisousharmonique sur $\Omega$ telle que $v \geq-1$ et $\Omega^{\prime}=\{v<0\} \subset \subset \Omega$. Soit $K$ un compact de $\Omega^{\prime}$. Si $C_{K}=-\sup _{K} v$, alors pour tout $1 \leq s \leq p$ on a

$$
\int_{K} T \wedge\left(d d^{c} u\right)^{p} \leq C_{K}^{-s} \int_{\Omega^{\prime}} T \wedge\left(d d^{c} v\right)^{s} \wedge\left(d d^{c} u\right)^{p-s} .
$$

Démonstration du théorème 2.7. Pour la démonstration on reprend des techniques de [BM-EM97] pour donner une estimation de la masse d'un courant en fonction de la masse de ses tranches. 
Sans perte de généralité on suppose que la masse totale des tranches $\left\langle T, \pi_{1}, z_{1}\right\rangle$ et $\left\langle T, \pi_{2}, z_{2}\right\rangle$ de $T$ est bornée par une constante $c>0$ pour tout $z_{1} \in K_{1}$ et $z_{2} \in K_{2}$. (Pour cela il suffit de prendre les ensembles

$$
\begin{aligned}
& A_{m}=\left\{z_{1} \in K_{1} ;\left\|\left\langle T, \pi_{1}, z_{1}\right\rangle\right\| \leq m\right\}, \\
& B_{m}=\left\{z_{2} \in K_{2} ;\left\|\left\langle T, \pi_{2}, z_{2}\right\rangle\right\| \leq m\right\} .
\end{aligned}
$$

Pour tout $m \in \mathbb{N}$, ces ensembles sont des boréliens qui recouvrent respectivement $K_{1}$ et $K_{2}$. Donc il existe $m \in \mathbb{N}$ tel que $A_{m}$ et $B_{m}$ sont non polaires. Ils contiennent donc chacun un compact non polaire.) On considère les fonctions extrémales de $K_{1}$ et $K_{2}$,

$$
\begin{aligned}
& u_{1}^{*}\left(z_{1}\right)=\sup _{u \in \operatorname{SH}(\mathbb{C})}\left\{u\left(z_{1}\right) ; u\left(z_{1}\right) \leq a+\log \left(1+\left|z_{1}\right|\right) \text {, et } u \leq-1 \text { sur } K_{1}\right\}, \\
& u_{2}^{*}\left(z_{2}\right)=\sup _{u \in \operatorname{SH}(\mathbb{C})}\left\{u\left(z_{2}\right) ; u\left(z_{2}\right) \leq a+\log \left(1+\left|z_{2}\right|\right) \text {, et } u \leq-1 \text { sur } K_{2}\right\} .
\end{aligned}
$$

Comme $K_{1}$ et $K_{2}$ sont des compacts non polaires, il existe des constantes $A, s, t>0$ telles que

$$
\begin{aligned}
\max \left(-1, \log \left(\frac{1+\left|z_{1}\right|}{s}\right)\right) & \leq u_{1}^{*}\left(z_{1}\right) \leq A+\log \left(1+\left|z_{1}\right|\right), \\
\max \left(-1, \log \left(\frac{1+\left|z_{2}\right|}{t}\right)\right) \leq u_{2}^{*}\left(z_{2}\right) & \leq A+\log \left(1+\left|z_{2}\right|\right) .
\end{aligned}
$$

Soit $R>0$ assez grand. On pose

$$
v_{R}\left(z_{1}, z_{2}\right)=\frac{u_{1}^{*}\left(z_{1}\right)-A}{\log (1+R)}+\frac{u_{2}^{*}\left(z_{2}\right)-A}{\log (1+R)}-2 .
$$

La fonction $v$ est psh et $\Omega_{R}^{\prime}=\left\{v_{R}<0\right\} \subset \subset \mathbb{C}^{2}$. Il est facile de démontrer que $K=\overline{B(0, R)} \subset \Omega^{\prime}$ et que $C_{K}=-\sup _{K} v_{R}=O(1 / \log (1+R))$. On pose $u\left(z_{1}, z_{2}\right)=|z|^{2} / R^{2}-1$. En appliquant le lemme précédent et la formule de tranchage on a

$$
\begin{aligned}
\frac{1}{R^{2}} \int_{B(0, R)} T \wedge \beta & \leq c_{K}^{-1} \int_{\Omega^{\prime}} T \wedge d d^{c} v_{R} \\
& \leq C \int_{K_{1}}\left\langle T, \pi_{1}, z_{1}\right\rangle(1) d d^{c} u_{1}^{*}+C \int_{K_{2}}\left\langle T, \pi_{2}, z_{2}\right\rangle(1) d d^{c} u_{2}^{*} \leq M,
\end{aligned}
$$

ce qui démontre que $T$ est algébrique.

3. Courants de Liouville. Un espace analytique complexe est dit de Liouville si les seules fonctions holomorphes bornées sur cet espace sont les constantes. Par le théorème de Liouville classique, $\mathbb{C}$ vérifie cette propriété, l'espace $\mathbb{C}^{n}$, ainsi que les graphes au-dessus de $\mathbb{C}^{n}$ (de la forme $\left\{(z, w) ; w=f(z), z \in \mathbb{C}^{n}\right\}$ où $f$ est une application holomorphe sur $\left.\mathbb{C}^{n}\right)$ sont 
aussi de Liouville. Plusieurs auteurs ([SI-WO81], [TA93], [KA96] et [TO96]) ont déja étudié les espaces ayant cette propriété. Néanmoins le problème de la caractérisation des espaces de Liouville reste encore ouvert.

Nous étendons cette propriété aux courants positifs fermés comme suit :

DÉfinition 3.1. Un courant positif fermé sur $\mathbb{C}^{n}$ est dit de Liouville si toute fonction holomorphe $f$ sur $\mathbb{C}^{n}$ bornée sur le support de $T$ vérifie $d d^{c}\left(|f|^{2} T\right)=0$.

ThÉORÈme 3.2. Soit $T$ un courant positif fermé sur $\mathbb{C}^{n}$ et u une fonction plurisousharmonique positive tels que

$$
\lim _{r \rightarrow+\infty} \frac{\nu_{u T}(r)}{\log r}=0
$$

Alors $d d^{c} u \wedge T=0$.

Corollaire 3.3. Tout courant algébrique est de Liouville.

La réciproque est fausse. Il suffit de prendre le courant d'intégration sur l'hypersurface de $\mathbb{C}^{2}$ définie par $\left\{z=\left(z_{1}, z_{2}\right) \in \mathbb{C}^{2} ; z_{2}=e^{z_{1}}\right\}$. Pour d'autres exemples on peut se référer à la thèse de M. T. Togni [TO96] et [DE79].

REMARQUE. Le théorème précédent généralise le résultat classique suivant : si $u$ est une fonction psh sur $\mathbb{C}^{n}$ telle que sa moyenne sur la boule de centre 0 et de rayon $r$, notée $\Lambda(u, r)$, vérifie $\Lambda(u, r)=o(\log r)$, alors $u$ est constante. Ce résultat se déduit du fait que l'application $r \mapsto \Lambda(u, r)$ est convexe croissante de $\log r$.

Pour démontrer le théorème 3.2 , on aura besoin de quelques résultats intermédiaires. On pose

$$
f(r)=\frac{1}{\pi^{p-1} r^{2 p-2}} \int_{B(0, r)} d d^{c} u \wedge T \wedge \beta^{p-1}=\nu\left(d d^{c} u \wedge T, 0, r\right) .
$$

Il est bien connu que la fonction $f$ est croissante et $\lim _{r \rightarrow 0} f(r)=0$. On note pour la suite

$$
h(r)=\nu_{u T}(r)=\frac{1}{\pi^{p} r^{2 p}} \int_{B(0, r)} u T \wedge \beta^{p} .
$$

Lemme 3.4. Il existe deux constantes $C^{\prime}, C^{\prime \prime}>0$ indépendantes de $R$ telles que

$$
\int_{1}^{R} \frac{f(t)}{t} d t \leq C^{\prime} h(2 R)+C^{\prime \prime}
$$


Démonstration. On a

$$
\begin{aligned}
\int_{1}^{R} \frac{f(t)}{t} d t= & \int_{1}^{R} \frac{d t}{t} \frac{1}{\pi^{p-1} t^{2 p-2}} \int_{B(0, t)} d d^{c} u \wedge T \wedge \beta^{p-1} \\
= & \int_{1}^{R} \frac{d t}{t} \frac{1}{\pi^{p-1} t^{2 p-2}} \int_{S(0, t)} d^{c} u \wedge T \wedge \beta^{p-1} \\
= & \frac{1}{2 \pi^{p-1}} \int_{B(0, R) \backslash B(0,1)} d|z|^{2} \wedge d^{c} u \wedge T \wedge \frac{\beta^{p-1}}{|z|^{2 p}} \\
= & \frac{1}{2 \pi^{p-1}} \int_{B(0, R) \backslash B(0,1)} d u \wedge d^{c}|z|^{2} \wedge T \wedge \frac{\beta^{p-1}}{|z|^{2 p}} \\
= & \frac{1}{2 \pi^{p-1}} \frac{1}{R^{2 p}} \int_{S(0, R)} u T \wedge \beta^{p-1} \wedge d^{c}|z|^{2} \\
& -\frac{1}{2 \pi^{p-1}} \int_{S(0,1)} u T \wedge \beta^{p-1} \wedge d^{c}|z|^{2} \\
& -\frac{1}{2 \pi^{p-1}} \int_{B(0, R) \backslash B(0,1)} u T \wedge d\left(\frac{\beta^{p-1} \wedge d^{c}|z|^{2}}{|z|^{2 p}}\right) .
\end{aligned}
$$

\section{Comme}

$$
\int_{B(0, R) \backslash B(0,1)} u T \wedge d\left(\frac{\beta^{p-1} \wedge d^{c}|z|^{2}}{|z|^{2 p}}\right)=\int_{B(0, R) \backslash B(0,1)} u T \wedge \alpha^{p} \geq 0,
$$

alors

$$
\int_{1}^{R} \frac{f(t)}{t} d t \leq F(R)=\frac{1}{2 \pi^{p-1} R^{2 p}} \int_{S(0, R)} u T \wedge \beta^{p-1} \wedge d^{c}|z|^{2}+C^{\prime \prime} .
$$

De plus,

$$
\begin{aligned}
\int_{R}^{2 R} \frac{F(s)}{s} d s & =\frac{1}{2 \pi^{p-1}} \int_{R}^{2 R} \frac{d s}{s}\left(\frac{1}{s^{2 p}} \int_{S(0, s)} u T \wedge \beta^{p-1} \wedge d^{c}|z|^{2}\right) \\
& =\frac{1}{2 \pi^{p-1}} \int_{R}^{2 R} \frac{d s}{s}\left(\int_{S(0, s)} u T \wedge \frac{\beta^{p-1} \wedge d^{c}|z|^{2}}{|z|^{2 p}}\right) \\
& =\frac{1}{2 \pi^{p-1}} \int_{B(0,2 R) \backslash B(0, R)} u T \wedge \frac{\gamma \wedge \beta^{p-1}}{|z|^{2 p+2}},
\end{aligned}
$$


avec $\gamma=d|z|^{2} \wedge d^{c}|z|^{2}$. On rappelle la relation classique

$$
\alpha^{p}=\frac{\beta^{p}}{|z|^{2 p}}-p \frac{\gamma \wedge \beta^{p-1}}{|z|^{2 p+2}}
$$

qui s'obtient par récurrence sur $p$. Il en résulte que

$$
\frac{\gamma \wedge \beta^{p-1}}{|z|^{2 p+2}} \leq \frac{\beta^{p}}{|z|^{2 p}}
$$

Le courant $u T$ est positif et $d d^{c}(u T) \geq 0$, donc la fonction $F$ est croissante. Il en résulte que

$$
\begin{aligned}
\int_{R}^{2 R} \frac{F(t)}{t} d t & \leq \frac{1}{2 \pi^{p-1}}=\int_{B(0,2 R) \backslash B(0, R)} u T \wedge \frac{\beta^{p}}{|z|^{2 p}} \\
& \leq \frac{1}{2 \pi^{p-1} R^{2 p}} \int_{B(0,2 R)} u T \wedge \beta^{p}=2^{2 p-1} \pi h(2 R) .
\end{aligned}
$$

Donc

$$
F(R) \leq \frac{2^{2 p-1} \pi}{\log 2} h(2 R)
$$

Corollaire 3.5. Avec les notations et les hypothèses précédentes, $d d^{c} u \wedge T=0$.

Démonstration. On a

$$
\int_{1}^{r} \frac{f(t)}{t} d t=o(\log r)
$$

comme $f$ est croissante,

$$
\int_{r}^{r^{2}} \frac{f(t)}{t} d t \geq f(r) \log r
$$

donc $f(r) \log r=o(\log r)$, ainsi $\lim _{r \rightarrow+\infty} f(r)=0$; ce qui donne $f \equiv 0$. Il en résulte que $d d^{c} u \wedge T=0$.

On se propose de donner des conditions suffisantes pour qu'un courant positif fermé soit de Liouville. On généralise le théorème de Sibony et Wong [SI-WO81], démontré pour les courants d'intégration sur un ensemble analytique, aux courants positifs fermés sur $\mathbb{C}^{n}$.

THÉORÈME 3.6. Soit $T$ un courant positif fermé sur $\mathbb{C}^{n}$ de bidimension $(p, p)$ et soit $u$ une fonction plurisousharmonique positive sur $\mathbb{C}^{n}$. On suppose que

$$
\lim _{R \rightarrow+\infty} \frac{M(u, R) N_{T}(R)}{(\log R)^{2}}=0, \quad \text { ò̀ } \quad M(u, R)=\sup _{|z| \leq R} u(z) .
$$

Alors $d d^{c} u \wedge T=0$. 
Démonstration. Il suffit de démontrer le théorème pour les fonctions psh de classe $\mathcal{C}^{\infty}$. En effet, il suffit de prendre les régularisées $u_{j}$ de $u$ avec un noyau régularisant radial positif et appliquer le théorème de BedfordTaylor : $d d^{c} u_{j} \wedge T$ converge faiblement vers $d d^{c} u \wedge T$. On pose

$$
f(r)=\int_{B(r)} T \wedge d d^{c} u \wedge \alpha^{p-1}
$$

Alors en appliquant le théorème de Stokes on a

$$
f(r)=\int_{S(r)} T \wedge d^{c} u \wedge \alpha^{p-1} .
$$

Si $0<s<r$ on a

$$
\begin{aligned}
\int_{s}^{r} \frac{f(t)}{t} d t= & \int_{s}^{r} \frac{d t}{t} \int_{S(t)} d^{c} u \wedge T \wedge \alpha^{p-1} \\
= & \frac{1}{2} \int_{B(r) \backslash B(s)} d \log |z|^{2} \wedge d^{c} u \wedge T \wedge \alpha^{p-1} \\
= & \frac{1}{2} \int_{B(r) \backslash B(s)} d u \wedge d^{c} \log |z|^{2} \wedge T \wedge \alpha^{p-1} \\
= & \frac{1}{2} \int_{S(r)} u T \wedge \alpha^{p-1} \wedge d^{c} \log |z|^{2}-\frac{1}{2} \int_{S(s)} u T \wedge \alpha^{p-1} \wedge d^{c} \log |z|^{2} \\
& -\frac{1}{2} \int_{B(r) \backslash B(s)} u T \wedge \alpha^{p} .
\end{aligned}
$$

Le dernier terme est négatif, le deuxième terme est borné. Pour le premier terme

$$
\int_{R}^{2 R} \frac{2 d r}{r} \int_{s}^{r} \frac{f(t)}{t} d t \geq 2 \log 2 \int_{s}^{R} \frac{f(t)}{t} d t
$$

et

$$
\begin{aligned}
\int_{R}^{2 R} \frac{2 d r}{r} \int_{s}^{r} \frac{f(t)}{t} d t= & \frac{1}{2} \int_{B(2 R) \backslash B(R)} u T \wedge \alpha^{p-1} d \log |z|^{2} \wedge d^{c} \log |z|^{2} \\
& -\frac{\log 2}{2} \int_{S(s)} u T \wedge \alpha^{p-1} \wedge d^{c} \log |z|^{2}-\int_{R}^{2 R} \frac{d r}{r} \int_{B(r) \backslash B(s)} u T \wedge \alpha^{p} \\
\leq & \frac{M(2 R)}{2} \int_{B(2 R) \backslash B(R)} T \wedge \alpha^{p-1} \wedge \frac{\gamma}{|z|^{4}}+C^{\prime}
\end{aligned}
$$




$$
\begin{aligned}
& \leq \frac{M(2 R)}{2} \int_{B(2 R) \backslash B(R)} T \wedge \frac{\beta^{p}}{|z|^{2 p}}+C^{\prime} \\
& =\frac{M(2 R)}{2 R^{2 p}} \int_{B(2 R)} T \wedge \beta^{p}+C^{\prime} \leq C \nu_{T}(2 R) M(2 R)+C^{\prime} .
\end{aligned}
$$

Donc

$$
\int_{s}^{R} \frac{f(t)}{t} d t \leq \frac{1}{2 \log 2} C \nu_{T}(2 R) M(2 R)+\frac{C^{\prime}}{2 \log 2} .
$$

Il en résulte que

$$
f(s) \log \frac{r}{s} \leq \int_{s}^{r} \frac{f(t)}{t} d t \leq \frac{C M(2 r) \nu_{T}(2 r)}{2 \log 2}+\frac{C^{\prime}}{2 \log 2},
$$

et

$$
f(s) \int_{s}^{R} \frac{\log \frac{r}{s}}{r} d r=\frac{f(s)}{2}\left(\log \frac{R}{s}\right)^{2} \leq \frac{C M(2 R)}{2 \log 2} N_{T}(2 R)+\frac{C^{\prime}}{2 \log 2} \log \frac{R}{s} .
$$

Il en résulte que

$$
f(s) \leq C \frac{M(2 R) N_{T}(2 R)}{\left(2 \log 2 \log \frac{R}{s}\right)^{2}}+\frac{C^{\prime}}{2 \log 2 \log \frac{R}{s}} .
$$

L'hypothèse faite sur $u$ et $T$ donne donc $f(s)=0$ et par suite $d d^{c} u \wedge T=0$.

\section{Théorème de K. Takegoshi pour les courants positifs fermés.} Sous les notations de la section précédente et du théorème 3.6, si $u$ est une fonction plurisousharmonique positive sur $\mathbb{C}^{n}$, nous avons donné une condition sur le courant positif fermé $T$ et sur la croissance de $u$ pour obtenir $d d^{c} u \wedge T=0$. K.Takegoshi [TA96] a, quant à lui, montré que si $T=[X]$ est le courant d'intégration sur un ensemble analytique complexe vérifiant

$$
\lim _{r \rightarrow+\infty} \int_{1}^{r} \frac{d t}{t \nu_{[X]}(t)}=+\infty
$$

alors les seules fonctions psh bornées sur $X$ sont les constantes. Dans cette section, on généralise ce dernier résultat aux courants positifs fermés. Pour cette généralisation, qui figure au théorème 4.3 , nous avons besoin de la proposition 4.1 et de l'estimation qui figure au théorème 4.2 .

Dans toute la suite, $\tau$ est une fonction exhaustive $\mathcal{C}^{\infty}$ et $\omega_{r}$ la mesure superficielle sur $\{\tau=r\}$ telle que pour tout $x \in\{\tau=r\}$, l'élément de volume $\beta^{n}(x) / n$ ! s'écrit

$$
\frac{\beta^{n}(x)}{n !}=d \tau(x) \wedge \omega_{r}(x)
$$


Proposition 4.1. Soient $T$ un courant positif fermé de bidimension $(1,1),\left(T_{j}\right)_{j}$ une suite de courants positifs fermés à coefficients continus qui converge faiblement vers $T$ et $u$ une fonction psh $\mathcal{C}^{\infty}$ positive sur $\mathbb{C}^{n}$, et soit $\tau$ une fonction psh $\mathcal{C}^{\infty}$ exhaustive. Alors

$\lim _{j \rightarrow+\infty} \int_{\{\tau=r\}} \operatorname{tr}\left(d \tau \wedge d^{c} u \wedge T_{j}\right) \omega_{r}=\int_{\{\tau<r\}} d d^{c} u \wedge T \quad$ pour presque tout $r>0$.

Démonstration. Pour tout réel $t$ positif on a

$$
\begin{aligned}
\int_{0}^{t} d r \int_{\{\tau=r\}} \operatorname{tr}\left(d \tau \wedge d^{c} u \wedge T_{j}\right) \omega_{r} & =\int_{\{\tau<t\}} \operatorname{tr}\left(d \tau \wedge d^{c} u \wedge T_{j}\right) d \tau \wedge \omega_{r} \\
& =\int_{\{\tau<t\}} d \tau \wedge d^{c} u \wedge T_{j}=\int_{0}^{t} d r \int_{\{\tau=r\}} d^{c} u \wedge T_{j},
\end{aligned}
$$

et par suite

$$
\int_{\{\tau=r\}} \operatorname{tr}\left(d \tau \wedge d^{c} u \wedge T_{j}\right) \omega_{r}=\int_{\{\tau=r\}} d^{c} u \wedge T_{j} \quad \text { pour presque tout } r>0 .
$$

D'autre part on a

$$
\lim _{j \rightarrow+\infty} \int_{\{\tau=r\}} d^{c} u \wedge T_{j}=\lim _{j \rightarrow+\infty} \int_{\{\tau<r\}} d d^{c} u \wedge T_{j}=\int_{\{\tau<r\}} d d^{c} u \wedge T
$$

en effet, soit $\left(\varphi_{\varepsilon}\right)_{0<\varepsilon<r}$ une famille de fonctions $\mathcal{C}^{\infty}$ à support dans $\{\tau<r\}$ telle que $0 \leq \varphi_{\varepsilon} \leq 1$ et $\varphi_{\varepsilon} \equiv 1$ sur $\{\tau<r-\varepsilon\}$; alors

$$
\begin{aligned}
& \left|\int_{\{\tau<r\}} d d^{c} u \wedge T_{j}-\int_{\{\tau<r\}} d d^{c} u \wedge T\right| \leq\left|\int_{\{\tau<r\}}\left(1-\varphi_{\varepsilon}\right) d d^{c} u \wedge T_{j}\right| \\
& +\left|\int_{\{\tau<r\}} \varphi_{\varepsilon} d d^{c} u \wedge T_{j}-\int_{\{\tau<r\}} \varphi_{\varepsilon} d d^{c} u \wedge T\right|+\left|\int_{\{\tau<r\}}\left(\varphi_{\varepsilon}-1\right) d d^{c} u \wedge T\right| .
\end{aligned}
$$

On a

$$
\begin{aligned}
\lim _{j \rightarrow+\infty} \mid \int_{\{\tau<r\}} \varphi_{\varepsilon} d d^{c} u \wedge T_{j}- & \int_{\{\tau<r\}} \varphi_{\varepsilon} d d^{c} u \wedge T \mid \\
& =\lim _{j \rightarrow+\infty}\left|\left\langle d d^{c} u \wedge T_{j}, \varphi_{\varepsilon}\right\rangle-\left\langle d d^{c} u \wedge T, \varphi_{\varepsilon}\right\rangle\right|=0 .
\end{aligned}
$$

De plus

$$
\lim _{\varepsilon \rightarrow 0} \int_{\{\tau<r\}}\left(\varphi_{\varepsilon}-1\right) d d^{c} u \wedge T=0,
$$

car $T$ est à coefficients mesures.

De même on a

$$
\lim _{\varepsilon \rightarrow 0} \int_{\{\tau<r\}}\left(\varphi_{\varepsilon}-1\right) d d^{c} u \wedge T_{j}=0
$$


Donc

$\lim _{j \rightarrow+\infty} \int_{\{\tau=r\}} \operatorname{tr}\left(d \tau \wedge d^{c} u \wedge T_{j}\right) \omega_{r}=\int_{\{\tau<r\}} d d^{c} u \wedge T \quad$ pour presque tout $r>0$.

THÉORÈme 4.2. Soient $T$ un courant positif fermé de bidimension $(1,1)$, $\left(T_{j}\right)_{j}$ une suite de courants positifs fermés à coefficients continus qui converge faiblement vers $T$ et $u$ une fonction psh $\mathcal{C}^{\infty}$ positive sur $\mathbb{C}^{n}$, et soit $\tau$ une fonction psh $\mathcal{C}^{\infty}$ exhaustive. Alors

$$
\left(\int_{\{\tau<r\}} d d^{c} u \wedge T\right)^{2} \leq\left(\int_{\{\tau<r\}} d d^{c} \tau \wedge T\right) \underset{j \rightarrow+\infty}{\lim _{\{\tau=r\}}} \int_{j} \operatorname{tr}\left(d u \wedge d^{c} u \wedge T_{j}\right) \omega_{r}
$$

pour presque tout $r>0$.

Démonstration. La démonstration se fait en deux étapes :

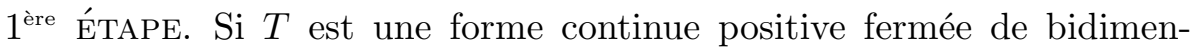
sion $(1,1)$, alors pour tout $z$ de $\mathbb{C}^{n}$ il existe un système de coordonnées au voisinage de ce point tel que

$$
T=\sum_{|I|=n-1} T_{I I} \sigma_{n-1} d z_{I} \wedge d \bar{z}_{I}
$$

Alors au point $z$ on a

$$
\begin{aligned}
d \tau \wedge d^{c} u \wedge T= & \sum_{j=1}^{n}\left(\frac{\partial \tau}{\partial z_{j}} d z_{j}+\frac{\partial \tau}{\partial \bar{z}_{j}} d \bar{z}_{j}\right) \\
& \wedge \sum_{k=1}^{n} \frac{i}{2}\left(\frac{\partial u}{\partial \bar{z}_{k}} d \bar{z}_{k}-\frac{\partial u}{\partial z_{k}} d z_{k}\right) \wedge \sum_{|I|=n-1} T_{I I} \sigma_{n-1} d z_{I} \wedge d \bar{z}_{I} \\
= & \sum_{j=1}^{n}\left(\frac{\partial \tau}{\partial z_{j}} \frac{\partial u}{\partial \bar{z}_{j}}+\frac{\partial \tau}{\partial \bar{z}_{j}} \frac{\partial u}{\partial z_{j}}\right) T_{I_{j} I_{j}} \frac{\beta^{n}}{n !}
\end{aligned}
$$

avec $I_{j}=\{1, \ldots, j-1, j+1, \ldots, n\}$, donc

$$
\begin{aligned}
\operatorname{tr}\left(d \tau \wedge d^{c} u \wedge T\right) & =\sum_{j=1}^{n}\left(\frac{\partial \tau}{\partial z_{j}} \frac{\partial u}{\partial \bar{z}_{j}}+\frac{\partial \tau}{\partial \bar{z}_{j}} \frac{\partial u}{\partial z_{j}}\right) T_{I_{j} I_{j}} \\
& =2 \operatorname{Re} \sum_{j=1}^{n} \frac{\partial \tau}{\partial z_{j}} \frac{\partial u}{\partial \bar{z}_{j}} T_{I_{j} I_{j}} .
\end{aligned}
$$

D'après l'inégalité de Cauchy-Schwarz on a

$$
\begin{aligned}
\left(\operatorname{tr}\left(d \tau \wedge d^{c} u \wedge T\right)\right)^{2} & \leq 4 \sum_{j=1}^{n} \frac{\partial \tau}{\partial z_{j}} \frac{\partial \tau}{\partial \bar{z}_{j}} T_{I_{j} I_{j}} \sum_{j=1}^{n} \frac{\partial u}{\partial z_{j}} \frac{\partial u}{\partial \bar{z}_{j}} T_{I_{j} I_{j}} \\
& \leq \operatorname{tr}\left(d u \wedge d^{c} u \wedge T\right) \operatorname{tr}\left(d \tau \wedge d^{c} \tau \wedge T\right)
\end{aligned}
$$


et par suite

$$
\operatorname{tr}\left(d \tau \wedge d^{c} u \wedge T\right) \leq \sqrt{\operatorname{tr}\left(d u \wedge d^{c} u \wedge T\right)} \sqrt{\operatorname{tr}\left(d \tau \wedge d^{c} \tau \wedge T\right)}
$$

en appliquant maintenant l'inégalité de Cauchy-Schwarz pour les intégrales on obtient

$\left(\int_{\{\tau=r\}} \operatorname{tr}\left(d \tau \wedge d^{c} u \wedge T\right) \omega_{r}\right)^{2} \leq \int_{\{\tau=r\}} \operatorname{tr}\left(d u \wedge d^{c} u \wedge T\right) \omega_{r} \int_{\{\tau=r\}} \operatorname{tr}\left(d \tau \wedge d^{c} \tau \wedge T\right) \omega_{r}$

$2^{\text {ème }}$ ÉTAPE. Soit $T$ est un courant positif fermé de bidimension $(1,1)$ et $\left(T_{j}\right)_{j}$ une suite de courants définis par des formes continues positives fermées qui converge faiblement vers $T$. D'après l'étape précédente, pour toute fonction psh $u$ et pour tout $j>0$ on a

$\left(\int_{\{\tau=r\}} \operatorname{tr}\left(d \tau \wedge d^{c} u \wedge T_{j}\right) \omega_{r}\right)^{2} \leq \int_{\{\tau=r\}} \operatorname{tr}\left(d u \wedge d^{c} u \wedge T_{j}\right) \omega_{r} \int_{\{\tau=r\}} \operatorname{tr}\left(d \tau \wedge d^{c} \tau \wedge T_{j}\right) \omega_{r}$

D'après la proposition 4.1 on a

$\lim _{j \rightarrow+\infty} \int_{\{\tau=r\}} \operatorname{tr}\left(d \tau \wedge d^{c} u \wedge T_{j}\right) \omega_{r}=\int_{\{\tau<r\}} d d^{c} u \wedge T \quad$ pour presque tout $r>0$ et

$\lim _{j \rightarrow+\infty} \int_{\{\tau=r\}} \operatorname{tr}\left(d \tau \wedge d^{c} \tau \wedge T_{j}\right) \omega_{r}=\int_{\{\tau<r\}} d d^{c} \tau \wedge T \quad$ pour presque tout $r>0$ donc

$$
\left(\int_{\{\tau<r\}} d d^{c} u \wedge T\right)^{2} \leq \int_{\{\tau<r\}} d d^{c} \tau \wedge T \underset{j \rightarrow+\infty}{\lim _{\{\tau=r\}}} \int_{j \rightarrow r} \operatorname{tr}\left(d u \wedge d^{c} u \wedge T_{j}\right) \omega_{r}
$$

pour presque tout $r>0$.

THÉORÈme 4.3. Soit $T$ un courant positif fermé de bidimension $(p, p)$, $1 \leq p \leq n-1$, sur $\mathbb{C}^{n}$. Soient $\tau: \mathbb{C}^{n} \rightarrow[\inf \tau,+\infty[$ une fonction psh exhaustive de classe $\mathcal{C}^{2}$ non bornée $g:(-\infty,+\infty) \rightarrow(0,+\infty)$ vérifiant les conditions (i) et (ii) suivantes :

(i) $\int_{\delta}^{+\infty} \frac{d t}{g(t)}=+\infty$

(ii) $\limsup _{r \rightarrow \infty} \frac{\int_{\{\tau<r\}} T \wedge\left(d d^{c} \tau\right)^{p}}{g(r)}<+\infty$.

Alors toute fonction u psh sur $\mathbb{C}^{n}$ positive vérifiant

$$
\limsup _{r \rightarrow \infty}\left(\sup _{\{\tau<r\} \cap \operatorname{Supp} T} u^{2}\right) \frac{\int_{\{\tau<r\}} T \wedge\left(d d^{c} \tau\right)^{p}}{g(r)}<+\infty
$$

vérifie $d d^{c} u \wedge T=0$. En particulier, toute fonction psh u bornée sur le support de $T$ vérifie $d d^{c} u \wedge T=0$. 
Démonstration. Soit $u$ une fonction psh, positive et de classe $\mathcal{C}^{\infty}$ sur $\mathbb{C}^{n}$. Supposons que $u$ vérifie

$$
\int_{\{\tau<r\}} d u \wedge d^{c} u \wedge T \wedge\left(d d^{c} \tau\right)^{p-1} \neq 0 \quad \forall r>\delta, \delta>0 .
$$

Posons

$$
H(r):=\int_{\{\tau<r\}} d u \wedge d^{c} u \wedge T \wedge\left(d d^{c} \tau\right)^{p-1}
$$

et soit $\left(T_{j}\right)_{j}$ une suite de courants positifs fermés $\mathcal{C}^{\infty}$ qui converge faiblement vers $T$. La formule de Stokes appliquée à cette intégrale donne

$$
\begin{aligned}
H(r) & =\lim _{j \rightarrow \infty}\left(\int_{\{\tau=r\}} u d^{c} u \wedge T_{j} \wedge\left(d d^{c} \tau\right)^{p-1}-\int_{\{\tau<r\}} u d d^{c} u \wedge T_{j} \wedge\left(d d^{c} \tau\right)^{p-1}\right) \\
& \leq \lim _{j \rightarrow \infty} \int_{\{\tau=r\}} u d^{c} u \wedge T_{j} \wedge\left(d d^{c} \tau\right)^{p-1}=\lim _{j \rightarrow \infty} \frac{1}{2} \int_{\{\tau=r\}} d^{c} u^{2} \wedge T_{j} \wedge\left(d d^{c} \tau\right)^{p-1} \\
& =\lim _{j \rightarrow \infty} \frac{1}{2} \int_{\{\tau<r\}} d d^{c} u^{2} \wedge T_{j} \wedge\left(d d^{c} \tau\right)^{p-1}=\frac{1}{2} \int_{\{\tau<r\}} d d^{c} u^{2} \wedge T \wedge\left(d d^{c} \tau\right)^{p-1} .
\end{aligned}
$$

D'après le théorème 4.2 on a

$$
\begin{aligned}
& {[H(r)]^{2} \leq \frac{1}{4}\left(\int_{\{\tau<r\}} d d^{c} u^{2} \wedge T \wedge\left(d d^{c} \tau\right)^{p-1}\right)^{2}} \\
& \leq \frac{1}{4} \int_{\{\tau<r\}} d d^{c} \tau \wedge T \wedge\left(d d^{c} \tau\right)^{p-1} \varliminf_{j \rightarrow+\infty} \int_{\{\tau=r\}} \operatorname{tr}\left(d u^{2} \wedge d^{c} u^{2} \wedge T_{j} \wedge\left(d d^{c} \tau\right)^{p-1}\right) \omega_{r} \\
& \leq \frac{1}{4} \int_{\{\tau<r\}} T \wedge\left(d d^{c} \tau\right)^{p} \varliminf_{j \rightarrow+\infty} \int_{\{\tau=r\}} 4 u^{2} \operatorname{tr}\left(d u \wedge d^{c} u \wedge T_{j} \wedge\left(d d^{c} \tau\right)^{p-1}\right) \omega_{r} \\
& \leq\left(\sup _{\{\tau<r\} \cap \operatorname{Supp} T} u^{2}\right) \int_{\{\tau<r\}} T \wedge\left(d d^{c} \tau\right)^{p} \underset{j \rightarrow+\infty}{\lim _{\{\tau=r\}}} \operatorname{tr}\left(d u \wedge d^{c} u \wedge T_{j} \wedge\left(d d^{c} \tau\right)^{p-1}\right) \omega_{r},
\end{aligned}
$$

pour presque tout $r>0$. D'après le lemme de Fatou on a, pour tous réels $s$ et $t$ positifs tels que $t>s \geq 0$,

$$
\begin{aligned}
& \int_{s}^{t} \underset{j \rightarrow+\infty}{\lim _{\{\tau=r\}}} \int_{\{r} \operatorname{tr}\left(d u \wedge d^{c} u \wedge T_{j} \wedge\left(d d^{c} \tau\right)^{p-1}\right) \omega_{r} d r \\
& \leq \lim _{j \rightarrow+\infty} \int_{s}^{t} d r \int_{\{\tau=r\}} \operatorname{tr}\left(d u \wedge d^{c} u \wedge T_{j} \wedge\left(d d^{c} \tau\right)^{p-1}\right) \omega_{r} \\
& \quad=\int_{\{s<\tau<t\}} d u \wedge d^{c} u \wedge T \wedge\left(d d^{c} \tau\right)^{p-1} \leq H(t)-H(s)=\int_{s}^{t} H^{\prime}(r) d r .
\end{aligned}
$$


$H^{\prime}$ existe presque partout car $H$ est croissante, donc

$$
\varliminf_{j \rightarrow+\infty} \int_{\{\tau=r\}} \operatorname{tr}\left(d u \wedge d^{c} u \wedge T_{j} \wedge\left(d d^{c} \tau\right)^{p-1}\right) \omega_{r} \leq H^{\prime}(r)
$$

pour presque tout $r>0$, et par suite

$$
[H(r)]^{2} \leq\left(\sup _{\{\tau<r\} \cap \operatorname{Supp} T} u^{2}\right) \int_{\{\tau<r\}} T \wedge\left(d d^{c} \tau\right)^{p} H^{\prime}(r)
$$

pour presque tout $r>0$, et puisque

$$
\int_{\{\tau<r\}} T \wedge\left(d d^{c} \tau\right)^{p} \leq C g(r),
$$

alors

$$
[H(r)]^{2} \leq C g(r) H^{\prime}(r) \quad \text { pour presque tout } r>0 .
$$

On en déduit alors que

$$
\int_{\delta}^{+\infty} \frac{1}{g(r)} d r \leq C \int_{\delta}^{+\infty} \frac{H^{\prime}(r)}{H^{2}(r)} d r \leq \frac{C}{H(\delta)}<+\infty .
$$

Ceci contredit les hypothèses du théorème, donc

$$
\int_{\{\tau<r\}} d u \wedge d^{c} u \wedge T \wedge\left(d d^{c} \tau\right)^{p-1}=0,
$$

alors

$$
d u \wedge d^{c} u \wedge T \wedge\left(d d^{c} \tau\right)^{p-1}=0 .
$$

Conséquences et optimalité des résultats. Dans ce paragraphe, nous déduisons des extensions des résultats déjà trouvés pour les ensembles analytiques ([SI-WO81] et [TA93]) aux courants positifs fermés. Nous étudions ensuite l'optimalité de ces résultats.

THÉORÈME 4.4. Soit $T$ un courant positif fermé de bidimension $(p, p)$, $1 \leq p \leq n-1$, sur $\mathbb{C}^{n}$ tel que

$$
\int_{\delta}^{+\infty} \frac{d t}{t \nu_{T}(t)}=+\infty
$$

Alors toute fonction psh u bornée sur le support de $T$ vérifie $d d^{c} u \wedge T=0$.

Démonstration. Soit $\tau(z)=\log \left(1+|z|^{2}\right)$, soit $\varphi$ la fonction psh exhaustive sur $\mathbb{C}^{n}$ définie par $\varphi(z)=1+\|z\|^{2}$, et posons $B_{\varphi}(r)=\{\varphi<r\}$ et 


$$
\begin{aligned}
& S_{\varphi}(r)=\{\varphi=r\} . \text { Alors } \\
& \begin{aligned}
\int_{B_{\varphi}(r)} T \wedge\left(d d^{c} \tau\right)^{p} & =\int_{S_{\varphi}(r)} T \wedge d^{c} \tau \wedge\left(d d^{c} \tau\right)^{p-1} \\
& \left.\left.=\int_{S_{\varphi}(r)} T \wedge d^{c} \log (1+\|z\|)^{2}\right) \wedge\left(d d^{c} \log (1+\|z\|)^{2}\right)\right)^{p-1} \\
& =\frac{1}{r^{p}} \int_{S_{\varphi}(r)} T \wedge \beta^{p-1} \wedge d^{c}\|z\|^{2}=\frac{1}{r^{p}} \int_{B_{\varphi}(r)} T \wedge \beta^{p} .
\end{aligned}
\end{aligned}
$$

On remplace dans l'équation précédente $r$ par $e^{r}$; alors

$$
\begin{aligned}
\int_{\{\tau<r\}} T \wedge\left(d d^{c} \tau\right)^{p} & =\frac{1}{e^{p r}} \int_{\left\{\|z\|^{2}<e^{r}-1\right\}} T \wedge \beta^{p}=\frac{\left(e^{r}-1\right)^{p}}{e^{p r}} \nu_{T}\left(\sqrt{e^{r}-1}\right) \\
& \leq \nu_{T}\left(\sqrt{e^{r}-1}\right) .
\end{aligned}
$$

En posant $g(t)=\nu_{T}\left(e^{t}\right)$ et en remarquant que

$$
\int_{\delta}^{+\infty} \frac{d t}{t \nu_{T}(t)}=\int_{\log \delta}^{+\infty} \frac{d t}{\nu_{T}\left(e^{t}\right)},
$$

le résultat de ce théorème se déduit alors du théorème 4.3.

Corollaire 4.5. Soit $T$ un courant positif fermé de bidimension $(p, p)$ sur $\mathbb{C}^{n}$ tel que

$$
\limsup _{r \rightarrow+\infty} \frac{N_{T}(r)}{(\log r)^{2}}<+\infty .
$$

Alors toute fonction psh $u$ bornée sur le support de $T$ est telle que $d d^{c} u \wedge T=0$.

Ce corollaire généralise le théorème de N. Sibony et P. M. Wong [SIWO81] pour les courants positifs fermés.

Démonstration du corollaire 4.5. Soit $T$ un courant positif fermé de bidimension $(p, p)$ dans $\mathbb{C}^{n}$ tel que

$$
\limsup _{r \rightarrow+\infty} \frac{N_{T}(r)}{(\log r)^{2}}<+\infty .
$$

Alors il existe une constante $C>0$ telle que pour tout $r>0$ on a

$$
\frac{\nu_{T}(r)}{\log r} \leq \frac{\int_{r}^{r^{2}} \frac{\nu_{T}(t)}{t} d t}{(\log r)^{2}}<C
$$

donc

$$
\frac{1}{r \log r}<\frac{C}{r \nu_{T}(r)}
$$


et par suite

$$
\int_{\delta}^{+\infty} \frac{1}{r \nu_{T}(r)} d r=+\infty
$$

D'après le théorème 4.4 toute fonction psh bornée sur le support de $T$ vérifie $d d^{c} u \wedge T=0$.

Dans la suite on donne un exemple de courant positif fermé tel que

$$
\int_{\delta}^{+\infty} \frac{1}{r \nu_{T}(r)} d r=+\infty \quad \text { et } \quad \limsup _{r \rightarrow+\infty} \frac{N_{T}(r)}{(\log r)^{2}}=+\infty
$$

Cet exemple montre que le corollaire précédent n'implique pas le théorème 4.4 .

EXEMPLE. Soit

$$
\varphi(z)=\sup \left(\log ^{2}\|z\|(\log (\log \|z\|)-1 / 2), 1\right) .
$$

Alors $\varphi$ est psh car

$$
\varphi(z)=\sup (f(\log \|z\|), 1) \quad \text { avec } \quad f: t \mapsto t^{2}(\log t-1 / 2),
$$

qui est convexe croissante pour $t>1$. Donc $T:=d d^{c} \varphi$ est un courant positif fermé. Pour $r$ assez grand on a

$$
\nu_{T}(r)=\frac{\partial \lambda(\varphi, 0, r)}{\partial \log r}=r \frac{\partial \lambda(\varphi, 0, r)}{\partial r}=r \frac{\partial\left((\log r)^{2}(\log (\log r)-1 / 2)\right)}{\partial r} ;
$$

alors

$$
\nu_{T}(r)=2 \log r \log (\log r)
$$

et

$$
\int_{\delta}^{+\infty} \frac{1}{r \nu_{T}(r)} d r=\frac{1}{2} \int_{\delta}^{+\infty} \frac{d r}{r \log r \log (\log r)}=+\infty
$$

Or on a

$$
\int_{\delta}^{r} \frac{\nu_{T}(t)}{t} d t=(\log r)^{2}(\log (\log r)-1 / 2)+C_{1}, \quad C_{1} \text { est une constante, }
$$

donc

$$
\limsup _{r \rightarrow+\infty} \frac{N_{T}(r)}{(\log r)^{2}}=+\infty .
$$

Exemple de courants de Liouville ne vérifiant pas le théorème 4.4. Nous allons nous intéresser au courant d'intégration sur l'ensemble analytique $M=\left\{(z, w) \in \mathbb{C}^{2} ; w=e^{-i z^{2}}\right\}$, qui est bien de Liouville comme graphe d'une fonction holomorphe (cf. [TO96]). 
Posons $\varphi(z)=e^{-i z^{2}}$ et $M(r)=\left\{z \in \mathbb{C} ;|z|^{2}+\left|e^{-i z^{2}}-1\right|^{2}<r^{2}\right\}$. Alors pour $r>2$ on a

$$
\begin{aligned}
\operatorname{Vol}(B(r) \cap M) & =\int_{M(r)}\left(1+\left|\varphi^{\prime}(z)\right|^{2}\right) d \lambda(z) \\
& \geq \int_{\{|z|<r / 2\} \cap\left\{\left|e^{-i z^{2}}\right|<r / 2-1\right\}}\left|\varphi^{\prime}(z)\right|^{2} d \lambda(z),
\end{aligned}
$$

ce qui permet d'obtenir après calculs la minoration suivante pour $r>2$ :

$$
\operatorname{Vol}(B(r) \cap M) \geq \frac{r^{2}}{8} \frac{2 \log (r / 2-1)-1}{(\log (r / 2-1))^{2}}(r / 2-1)^{2} .
$$

Or le second terme se comporte à l'infini comme $r^{4} / \log r$, il résulte donc que

$$
\int_{\delta}^{+\infty} \frac{1}{r \nu_{[M]}(r)} d r=\int_{\delta}^{+\infty} \frac{r}{\operatorname{Vol}(B(r) \cap M)} d r \leq C \int_{\delta}^{+\infty} \frac{\log r}{r^{3}} d r<+\infty .
$$

5. Courants positifs fermés à supports tubulaires. Soit $T$ un courant positif fermé sur $\mathbb{C}^{n}$ à support dans $\{|P| \leq 1\}$, où $P$ est un polynôme non constant sur $\mathbb{C}^{n}$ à valeurs dans $\mathbb{C}$. Alors $T$ est algébrique d'après le corollaire 2.5. On se propose dans ce paragraphe de donner un théorème de support pour ce type de courants $T$; pour ceci nous aurons besoin des lemmes 5.1 et 5.2 suivants :

LEMme 5.1. Soient $f$ une fonction holomorphe non constante sur $\mathbb{C}^{n} \grave{a}$ valeurs dans $\mathbb{C}, U$ un ouvert de $\mathbb{C}^{n}$ et $T$ un courant positif fermé de bidegré $(1,1)$ sur $U$. On suppose qu'il existe une mesure $\mu_{U}$ sur $f(U) \subset \mathbb{C}$ telle que

$$
T=\int_{f(U)}[f=t] d \mu_{U}(t)
$$

i.e. pour toute forme $\psi \in \mathcal{D}_{n-1, n-1}(U)$ on a

$$
\langle T, \psi\rangle=\int_{f(U)}\left(\int_{\{f=t\}} \psi\right) d \mu_{U}(t) .
$$

Alors $\mu_{U}$ est positive et elle est unique.

Démonstration. Soit $\chi$ une fonction continue sur $\mathbb{C}$ et à support compact dans $f(U)$, et soit $\beta \in \mathcal{D}_{n-1, n-1}(U)$ une forme fortement positive sur $U$ et strictement positive sur $f^{-1}(\operatorname{Supp} \chi)$. On définit une fonction $\chi_{1}$ continue à support compact dans $f(U)$ par

$$
\chi_{1}(t):=\chi(t)\left[\int_{\{f=t\}} \beta\right]^{-1}
$$


et soit $\psi$ la $(n-1, n-1)$-forme continue à support compact dans $U$ définie $\operatorname{par} \psi=\left(\chi_{1} \circ f\right) \beta$. Alors

$$
\begin{aligned}
\langle T, \psi\rangle & =\int_{f(U)}\left(\int_{\{f=t\}} \psi\right) d \mu_{U}(t) \\
& =\int_{f(U)}\left(\chi_{1}(t) \int_{\{f=t\}} \beta\right) d \mu_{U}(t)=\int_{f(U)} \chi(t) d \mu_{U}(t),
\end{aligned}
$$

d'où l'unicité et la positivité de $\mu_{U}$.

Lemme 5.2. Soit $T$ un courant positif fermé de bidegré $(1,1)$ sur un ouvert $U$ de $\mathbb{C}^{n}$ tel que $d z_{1} \wedge d \bar{z}_{1} \wedge T=0$. Alors il existe une mesure de Radon positive $\mu_{U}$ sur $\pi(U)$, avec $\pi: z \mapsto z_{1}$ la projection canonique, telle que

$$
T=\int_{\pi(U)}\left[z_{1}=t\right] d \mu_{U}(t)
$$

Démonstration. Soit $K$ un compact de $U$. Il existe une fonction psh $\varphi$ sur $U$ telle que

$$
T=d d^{c} \varphi=\sum_{j, k=1}^{n} \frac{\partial^{2} \varphi}{\partial z_{j} \partial \bar{z}_{k}} d z_{j} \wedge d \bar{z}_{k} \quad \text { sur un voisinage de } K .
$$

L'égalité $d z_{1} \wedge d \bar{z}_{1} \wedge T=0$ implique que pour tous $j, k \geq 2$ on a

$$
\frac{\partial^{2} \varphi}{\partial z_{j} \partial \bar{z}_{k}}=0
$$

De plus, d'après l'inégalité de Cauchy-Schwarz, pour tout $\varepsilon>0$ on a

$$
\frac{\partial^{2} \varphi}{\partial z_{j} \partial \bar{z}_{k}} \leq \varepsilon \frac{\partial^{2} \varphi}{\partial z_{j} \partial \bar{z}_{j}}+\frac{1}{\varepsilon} \frac{\partial^{2} \varphi}{\partial z_{k} \partial \bar{z}_{k}}
$$

On en déduit que $T$ s'écrit, au voisinage de $K$,

$$
T=\frac{\partial^{2} \varphi}{\partial z_{1} \partial \bar{z}_{1}} d z_{1} \wedge d \bar{z}_{1}
$$

On remarque de plus que $\partial^{2} \varphi / \partial z_{1} \partial \bar{z}_{1}$ est holomorphe par rapport à la variable $z_{k}$ pour tout $k \geq 2$, car

$$
\frac{\partial}{\partial \bar{z}_{k}}\left(\frac{\partial^{2} \varphi}{\partial z_{1} \partial \bar{z}_{1}}\right)=\frac{\partial}{\partial \bar{z}_{1}}\left(\frac{\partial^{2} \varphi}{\partial z_{1} \partial \bar{z}_{k}}\right)=0
$$

De même $\partial^{2} \varphi / \partial z_{1} \partial \bar{z}_{1}$ est antiholomorphe par rapport à la variable $z_{k}$ pour tout $k \geq 2$ puisque

$$
\frac{\partial}{\partial z_{k}}\left(\frac{\partial^{2} \varphi}{\partial z_{1} \partial \bar{z}_{1}}\right)=\frac{\partial}{\partial z_{1}}\left(\frac{\partial^{2} \varphi}{\partial z_{k} \partial \bar{z}_{1}}\right)=0 .
$$


Il s'ensuit que $\partial^{2} \varphi / \partial z_{1} \partial \bar{z}_{1}$ ne dépend pas des variables $z_{2}, \ldots, z_{n}$. Soit $\psi \in$ $\mathcal{D}_{n-1, n-1}(K)$; alors

$$
\begin{aligned}
\langle T, \psi\rangle & =\int_{U} \frac{\partial^{2} \varphi}{\partial z_{1} \partial \bar{z}_{1}} d z_{1} \wedge d \bar{z}_{1} \wedge \psi \\
& =\int_{\pi(U)}\left(\int_{\left\{z_{1}\right\} \times \mathbb{C}^{n-1}} \psi\right) \frac{\partial^{2} \varphi}{\partial z_{1} \partial \bar{z}_{1}} d z_{1} \wedge d \bar{z}_{1}=\int_{\pi(U)}\left(\int_{\left\{z_{1}=t\right\}} \psi\right) d \mu(t) .
\end{aligned}
$$

ThÉORÈme 5.3. Soit $T$ un courant positif fermé de bidegré $(1,1)$ sur $\mathbb{C}^{n}$. On suppose qu'il existe un polynôme non constant $P$ sur $\mathbb{C}^{n}$ tel que $\operatorname{Supp} T \subset$ $\left\{z \in \mathbb{C}^{n} ;|P(z)|<1\right\}$. On note $V$ (resp. $V_{i}$ ) l'espace des composantes irréductibles des différentes fibres $P^{-1}(t)$ dans $\mathbb{C}^{n}, t \in \mathbb{C} \backslash\left\{t_{1}, \ldots, t_{N}\right\}$ (resp. de $P^{-1}\left(t_{i}\right)$, où les $t_{i}$ sont les valeurs critiques de $\left.P\right)$. Alors il existe une unique mesure positive $\mu$ sur $\bar{V}=V \amalg \coprod_{i} V_{i}$ telle que

$$
T=\int_{v \in \bar{V}}\left[P^{-1}(t)\right]_{v} d \mu(v)
$$

où $\left[P^{-1}(t)\right]_{v}$ désigne le courant d'intégration sur la composante irréductible $v$ du sous-ensemble analytique $P^{-1}(t)$. C'est-à-dire que pour toute forme différentielle $\psi \in \mathcal{D}_{n-1, n-1}\left(\mathbb{C}^{n}\right)$,

$$
\langle T, \psi\rangle=\int_{v \in \bar{V}}\left\langle\left[P^{-1}(t)\right]_{v}, \psi\right\rangle d \mu(v) .
$$

Démonstration. $T$ est algébrique, donc $d P \wedge d \bar{P} \wedge T=0$. Soit $a \in$ $\mathbb{C}^{n} \backslash\{d P=0\}$. Alors il existe $i \in\{1, \ldots, n\}$ tel que $\partial P / \partial z_{i}(a) \neq 0$. On suppose que $i=1$; alors il existe un voisinage $U$ de $a$ sur lequel $F(z)=\left(P(z), z_{2}, \ldots, z_{n}\right)$ est un biholomorphisme, donc

$$
d P \wedge d \bar{P} \wedge T=F^{*}\left(d z_{1} \wedge d \bar{z}_{1} \wedge F_{*} T\right)=0 \quad \text { sur } U
$$

d'où

$$
d z_{1} \wedge d \bar{z}_{1} \wedge F_{*} T=0 \quad \text { sur } F(U) .
$$

Pour toute forme $\psi \in \mathcal{D}_{n-1, n-1}(U)$, on a

$$
\langle T, \psi\rangle=\left\langle T, F^{*} F_{*} \psi\right\rangle=\left\langle F_{*} T, F_{*} \psi\right\rangle,
$$

donc d'après les lemmes 5.1 et 5.2 , il existe une unique mesure de Radon positive $\mu_{U}$ sur $\pi(F(U))=P(U)$ telle que

$$
\begin{aligned}
\langle T, \psi\rangle & =\int_{P(U)}\left\langle\left[z_{1}=t\right], F_{*} \psi\right\rangle d \mu_{U}(t) \\
& =\int_{P(U)}\left\langle F^{*}\left[z_{1}=t\right], \psi\right\rangle d \mu_{U}(t)=\int_{P(U)}\langle[P=t], \psi\rangle d \mu_{U}(t) \quad \text { sur } U .
\end{aligned}
$$


On munit $\mathbb{C}^{n} \backslash P^{-1}\left(\left\{t_{1}, \ldots, t_{N}\right\}\right)$ de la relation d'équivalence $\sim$ telle que $z \sim z^{\prime}$ si et seulement si $z$ et $z^{\prime}$ sont dans une même composante connexe de $P^{-1}(P(z))$. L'espace quotient $\left(\mathbb{C}^{n} \backslash P^{-1}\left(\left\{t_{1}, \ldots, t_{N}\right\}\right)\right) / \sim$ muni de la topologie induite s'identifie à l'espace $V$ des composantes connexes des différentes $P^{-1}(t), t \in \mathbb{C} \backslash\left\{t_{1}, \ldots, t_{N}\right\}$, où les $t_{i}$ sont les valeurs critiques de $P$. Considérons le revêtement $P_{\mid V}: V \rightarrow \mathbb{C} \backslash\left\{t_{1}, \ldots, t_{N}\right\}$. En faisant varier $a$ sur la même composante connexe $v$ de $P^{-1}(t)$, puis varier $t$ sur un voisinage de $P(a)$, l'unicité des mesures $\mu_{U}, U$ recouvrant un voisinage saturé assez petit de $v$ dans $\mathbb{C}^{n} \backslash P^{-1}\left(\left\{t_{1}, \ldots, t_{N}\right\}\right)$, entraîne que la famille $\left(\mu_{U}\right)_{U}$ se recolle bien en une seule mesure sur ce voisinage. Ceci permet de définir une unique mesure $\nu$ sur la composante connexe de $V$ contenant $v$. Ainsi l'égalité précédente entraîne que

$$
T_{\mid \mathbb{C}^{n} \backslash P^{-1}\left(\left\{t_{1}, \ldots, t_{N}\right\}\right)}=\int_{v \in V}\left[P^{-1}(t)\right]_{v} d \nu(v) .
$$

Par le théorème de prolongement de Skoda-El Mir, le prolongement trivial $\widetilde{T}$ de $T_{\mid \mathbb{C}^{n} \backslash P^{-1}\left(\left\{t_{1}, \ldots, t_{N}\right\}\right)}$ existe. Le courant $T-\widetilde{T}$ étant positif fermé sur $\mathbb{C}^{n}$ de dimension $n-1$ et à support dans les composantes de dimension $n-1$ de $\{d P=0\}$, d'après le théorème de support de $\mathrm{H}$. Federer on a

$$
T-\widetilde{T}=\sum_{\text {fini }} c_{i}\left[X_{i}\right], \quad c_{i}>0 .
$$

De plus

$$
d P \wedge d \bar{P} \wedge(T-\widetilde{T})=d P \wedge d \bar{P} \wedge \sum_{\text {fini }} c_{i}\left[X_{i}\right]=0,
$$

et puisque les $\left[X_{i}\right]$ sont algébriques, $P$ est constant sur $X_{i}$. On a donc $X_{i} \subset$ $\left\{P^{-1}\left(t_{i}\right)\right\}$. Le courant $\left[X_{i}\right]$ s'écrit alors

$$
\left[X_{i}\right]=\sum_{1 \leq j \leq k(i)} b_{i, j}\left[P^{-1}\left(t_{i}\right)\right]_{v_{j}},
$$

où $b_{i, j} \geq 0$ et $\left[P^{-1}\left(t_{i}\right)\right]_{v_{j}}$ décrit les courants d'intégration sur les composantes irréductibles de $\left\{P^{-1}\left(t_{i}\right)\right\}$. Finalement, on a

$$
\begin{aligned}
T & =\widetilde{T}+\sum_{\text {fini }} a_{i, j}\left[P^{-1}\left(t_{i}\right)\right]_{v_{j}} \\
& =\int_{v \in V}\left[P^{-1}(t)\right]_{v} d \nu(v)+\sum_{\text {fini }} a_{i, j}\left[P^{-1}\left(t_{i}\right)\right]_{v_{j}},
\end{aligned}
$$

où les $a_{i, j}$ sont positifs. La mesure $\mu$ du théorème est donnée par

$$
\mu=\nu+\sum a_{i, j} \delta_{v_{j}}
$$

où $\delta_{v_{j}}$ est la mesure de Dirac en $v_{j}$. 
Remarque. On rappelle qu'un courant positif fermé $T$ est dit extrémal si, dès que $T=T_{1}+T_{2}$ où les $T_{j}$ sont positifs fermés non nuls, alors il existe $\lambda_{1}, \lambda_{2} \geq 0$ tels que $T=\lambda_{1} T_{1}=\lambda_{1} T_{2}$. Par exemple le courant d'intégration sur un sous-ensemble analytique complexe irréductible de $\mathbb{C}^{n}$ est un courant extrémal. Il résulte du théorème 5.3 qu'un courant positif fermé extrémal de type $(1,1)$ dont le support est contenu dans $\{|P|<1\}$ est le courant d'intégration sur un sous-ensemble algébrique irréductible.

\section{Bibliographie}

[AU95] B. Aupetit, A geometrical characterization of algebraic varieties of $\mathbb{C}^{2}$, Proc. Amer. Math. Soc. 123 (1995), 3323-3327.

[BT82] E. Bedford and B. A. Taylor, A new capacity for plurisubharmonic functions, Acta Math. 149 (1982), 1-40.

[BM-EM97] H. Ben Messaoud et H. El Mir, Tranchage et prolongement des courants positifs fermés, Math. Ann. 307 (1997), 473-487.

[BL89] M. Blel, Cône tangent à un courant positif fermé de type $(1,1), \mathrm{C}$. R. Acad. Sci. Paris 309 (1989), 543-546.

[BL92] —, Thèse d'État, Faculté des Sciences de Monastir, 1992.

[BL-DE-MO90] M. Blel, J.-P. Demailly et M. Mouzali, Sur l'existence du cône tangent à un courant positif fermé, Ark. Mat. 28 (1990), 231-248.

[DE79] J.-P. Demailly, Fonctions holomorphes à croissance polynômiale sur la surface d'équation $e^{x}+e^{y}=1$, Bull. Sci. Math. 103 (1979), 179-191.

[DE85] - Mesures de Monge-Ampère et caractérisation des variétés algébriques affines, Mém. Soc. Math. France 19 (1985).

[DE00] - Algebraic Geometry, 2000.

[EM84] H. El Mir, Sur le prolongement des courants positifs fermés, Acta Math. 153 (1984), 1-45.

[FO-SI95] J. E. Fornæss and N. Sibony, Oka's inequality for currents and applications, Math. Ann. 301 (1995), 399-419.

[KA96] H. Kaneko, Liouville theorems based on symmetric diffusions, Bull. Soc. Math. France 124 (1996), 545-557.

[LE-GR86] P. Lelong and L. Gruman, Entire Functions of Several Complex Variables, Grundlehren Math. Wiss. 282, Springer, 1986.

[MI00] S. K. Mimouni, Théorèmes de type Liouville pour les courants positifs fermés C. R. Acad. Sci. Paris 331 (2000), 611-616.

[MO-SH-SI81] R. Molzon, B. Shifman and N. Sibony, Average growth estimate for hyperplane sections of entire analytic sets, Math. Ann. 257 (1981), 4359.

[RA96] G. Raby, Tranchage des courants positifs fermés et équation de LelongPoincaré, J. Math. Pures Appl. 75 (1996), 189-209.

[RO80] L. I. Ronkin, Liouville's theorems for functions holomorphic on the zero set of a polynomial, Ukrainian Math. J. 31 (1980), 462-464.

[SI-WO81] N. Sibony and P. M. Wong, Some remarks on the Casorati-Weierstrass theorem, Ann. Polon. Math. 39 (1981), 165-174.

[SI74] Y. T. Siu, Analyticity of sets associated to Lelong numbers and the extension of closed positive currents, Invent. Math. 27 (1974), 53-156. 
[SK82] H. Skoda, Prolongement des courants positifs fermés de masse finie, ibid. 66 (1982), 361-376.

[ST64] W. Stoll, The growth of the area of a transcendental analytic set I, II, Math. Ann. 165 (1964), 47-78, 144-170.

[TA90] K. Takegoshi, Energy estimates and Liouville theorems for harmonic maps, Ann. Sci. École Norm. Sup. 23 (1990), 563-592.

[TA93] -, A Liouville theorem on an analytic space, J. Math. Soc. Japan 45 (1993), 301-311.

[TO96] M. T. Togni, Sur les espaces de Liouville, Thèse d'Université, Univ. de Bordeaux I, 1996.

M. Blel

Faculté des Sciences de Monastir

Département de mathématiques

5019 Monastir, Tunisie

E-mail: M.Blel@fsm.rnu.tn, mongib@ksu.edu.sa

S. K. Mimouni

Faculté des Sciences de Monastir

Département de mathématiques

5019 Monastir, Tunisie

E-mail: souad.khemiri@fsm.rnu.tn
G. Raby

UMR CNRS 6086

Groupes de Lie et Géométrie Mathématiques

Université de Poitiers

Téléport2-BP 30179

86962 Futuroscope Chasseneuil, France

E-mail: raby@mathlabo.univ-poitiers.fr

Reçu par la Rédaction le 16.6.2005

Révisé le 25.9.2005 